\title{
OPTIMAL DYNAMIC REINSURANCE
}

\author{
BY \\ DAVID C.M. Dickson AND HowARD R. WATERS
}

\begin{abstract}
We consider a classical surplus process where the insurer can choose a different level of reinsurance at the start of each year. We assume the insurer's objective is to minimise the probability of ruin up to some given time horizon, either in discrete or continuous time. We develop formulae for ruin probabilities under the optimal reinsurance strategy, i.e. the optimal retention each year as the surplus changes and the period until the time horizon shortens. For our compound Poisson process, it is not feasible to evaluate these formulae, and hence determine the optimal strategies, in any but the simplest cases. We show how we can determine the optimal strategies by approximating the (compound Poisson) aggregate claims distributions by translated gamma distributions, and, alternatively, by approximating the compound Poisson process by a translated gamma process.
\end{abstract}

\section{KEYWORDS}

Finite time ruin, reinsurance, dynamic strategy, translated gamma distribution, translated gamma process

\section{INTRODUCTION}

Reinsurance is an important mechanism by which an insurer can manage the financial risk of its operation. See, for example, the many references to reinsurance in Daykin et al. (1993). Many authors have considered the problem of determining the optimal level and/or type of reinsurance, where optimal is defined in terms of some stability criterion such as the variance of aggregate claims or the probability of ruin.

Centeno (1986) considered mixtures of excess loss and proportional reinsurance. She used the insurer's adjustment coefficient as a proxy for the (inverse of the) probability of ultimate ruin, so that a given combination of excess loss and proportional reinsurance is optimal if it maximises the insurer's adjustment coefficient, net of reinsurance. In 1986, algorithms for evaluating the probability of ruin were in their infancy, hence the attraction of using the adjustment coefficient. Since 1986, the situation has changed. 
The present authors, Dickson and Waters (1996), used numerical methods to determine the level of reinsurance which minimises the insurer's probability of ultimate ruin. We also investigated the effect of reinsurance on the probability of ruin in finite time.

A common feature of the studies mentioned above, and of almost all other studies in this area, is that the level and type of reinsurance is assumed to remain constant throughout the period being considered, which in many cases is infinite. In other words, the reinsurance strategy is static. Two relatively recent papers, Schmidli (2001) and Hipp and Vogt (2003), investigated dynamic reinsurance strategies for a fixed type of reinsurance, the former in relation to proportional and the latter in relation to excess loss reinsurance. In both these papers the level of reinsurance was allowed to change continuously so that, in mathematical terms, both papers deal with an optimal stochastic control problem in continuous time. Variations on this problem are considered in Schmidli (2002) and Promislow and Young (2005), where, in each case, the insurer can invest in risky and riskless assets.

In this paper we consider a classical (compound Poisson) model for the insurer's surplus. We assume that the insurer can change the type and/or level of reinsurance at the start of each year. We further assume that the insurer fixes a future time point and chooses the reinsurance arrangement each year which minimises the probability of ruin in the period up to that time point. Hence, at the start of each year, the optimal reinsurance arrangement will depend on the current level of the insurer's surplus and on the remaining time period, which decreases by one year each year. We study two cases for this problem, viz the probability of ruin in discrete time (at the end of each year only) and in continuous time.

Our problem is similar to the problems studied by Schmidli (2001) and Hipp and Vogt (2003) in that the reinsurance strategy is dynamic, but there are important differences. In their papers, the level of reinsurance can change continuously whereas in our model it can change only at the start of each year, which we consider more realistic. Mathematically, this means that we are studying a discrete time stochastic control problem, even in the case where, when we consider continuous time ruin, the underlying surplus process is a continuous time process. Another difference is that our optimality criterion is to minimise the probability of ruin up to some fixed time point, rather than in infinite time. A further difference, at least in principle, is that we can allow the insurer to change the type, as well as the level, of reinsurance each year. However, in our numerical examples in Section 7 the type of reinsurance is fixed and there would have been numerical difficulties if it had been allowed to change. Finally, the emphasis in this paper is on deriving formulae from which numerical results can be obtained.

We start in Section 2 by setting out our underlying model for the insurer's surplus process. Then, in Section 3, we use the Bellman Optimality Principle to derive recursive formulae which can, in principle, be used to determine optimal dynamic reinsurance strategies in discrete and in continuous time. We discuss the computational difficulties involved in obtaining numerical results from 
these formulae. In Section 4 we show how we can obtain numerical results, at least approximately, by replacing the distribution function (cdf) and density function (pdf) of a compound Poisson random variable by the cdf and pdf, respectively, of a translated gamma random variable, matched by moments. This is an idea which goes back at least to Seal (1978). In Section 5 we use a different approximation method to obtain numerical results: we replace the insurer's (net of reinsurance) compound Poisson aggregate claims process by a translated gamma process matched by moments. This is an idea which goes back to Dickson and Waters (1993). One of the secondary aims of this paper is to investigate the extent to which these two approximation procedures produce similar results. In Section 6 we discuss the computational procedures we have used to implement the formulae in Sections 4 and 5 and in Section 7 we present a selection of numerical results. In Section 8 we discuss some conclusions.

\section{THe Model}

Our study is based on the classical surplus process. Measuring time in years, we denote this process by $\{U(t)\}_{t \geq 0}$ where

$$
U(t)=u+c t-\sum_{i=1}^{N(t)} X_{i}
$$

where $u$ is the insurer's surplus at time $0, c$ is the rate of premium income per annum, $\{N(t)\}_{t \geq 0}$ is a Poisson process with parameter $\lambda$, and $\left\{X_{i}\right\}_{i=1}^{\infty}$ is a sequence of independent and identically distributed random variables with $X_{i}$ representing the amount of the $i$ th individual claim. We assume that $\operatorname{Pr}\left(X_{1}>0\right)=1$.

We now modify this process by introducing reinsurance. In this paper we consider reinsurance that applies to individual claims, rather than to aggregate claims. Thus, if $h$ denotes a reinsurance arrangement, we assume that for an individual claim of amount $x$, the insurer pays $h(x)$ such that $0 \leq h(x) \leq x$. We further assume that reinsurance premiums are payable continuously, and that the reinsurer pays its share of a claim as soon as that claim occurs.

We next assume that at the start of each year the insurer can determine the retention level for the coming year. Let $\theta_{i}$ denote the retention level in year $i$, i.e. from time $i-1$ to $i$. We will drop the subscript $i$ if we are discussing a generic reinsurance arrangement or if it is clear which year we are discussing. Let $U_{\theta}(n)$ denote the insurer's net (of reinsurance) surplus at time $n, n=0,1, \ldots$, given $\theta_{1}, \theta_{2}, \ldots, \theta_{n}$, where $U_{\theta}(0)=u$. Then for $t$ in $[0,1)$ we have

$$
U_{\theta_{n+1}}(n+t)=U_{\theta}(n)+c_{\theta_{n+1}} t-S_{\theta_{n+1}}(t)
$$

where $c_{\theta_{n+1}}$ denotes the insurer's net (of reinsurance) premium income per annum in year $n+1$ and $S_{\theta_{n+1}}(t)$ denotes the net aggregate claims in $[n, n+t]$, given reinsurance of amount $\theta_{n+1}$, so that $S_{\theta_{n+1}}(t)$ has a compound Poisson distribution. For a given value of $\theta$ and for $0 \leq t<1$, let 


$$
G_{\theta}(x, t)=\operatorname{Pr}\left(S_{\theta}(t) \leq x\right)
$$

be the cdf of $S_{\theta}(t)$, and for $x>0$ let $g_{\theta}(x, t)=\frac{\partial}{\partial x} G_{\theta}(x, t)$ denote the pdf.

We are interested in calculating finite time ruin probabilities for the net of reinsurance surplus process. Let $\psi_{\theta}(u, 1)$ denote the probability of ruin given initial surplus $u$, either at the end of the year or continuously through the year. We will use the same notation for ruin probabilities whether we are considering discrete or continuous time ruin. Next, let $\hat{\psi}(u, 1)$ denote the minimum value of $\psi_{\theta}(u, 1)$ over all admissible values of $\theta$. By admissible we mean that the net profit condition is satisfied, namely that $c_{\theta}>E\left[S_{\theta}(1)\right]$. Finally, let $\hat{\psi}(u, T)$ denote the minimum value of the probability of ruin over $T$ years, starting from initial surplus $u$, over all admissible values of $\theta$, where the value of $\theta$ can change from year to year.

\section{RECURSIVE FORMULAE}

In this section we present formulae for ruin probabilities over a $T$ year period, first in discrete time, then in continuous time. We then discuss the issues involved in applying these formulae. In discrete time, ruin is the event that the insurer's surplus, net of reinsurance, is below 0 for some $t, t=1,2, \ldots, T$, while in continuous time, ruin is the event that the insurer's surplus, net of reinsurance, is below 0 for some $t, 0<t \leq T$.

\subsection{Discrete time}

Let us consider the problem of minimising the probability of ruin in discrete time. As ruin occurs in the first year only if $S_{\theta}(1)>u+c_{\theta}$, we have

$$
\hat{\psi}(u, 1)=\inf _{\theta}\left(1-G_{\theta}\left(u+c_{\theta}, 1\right)\right)
$$

and the Bellman Principle tells us that for any initial surplus $u, \hat{\psi}(u, n)$ can be calculated recursively for $n=2,3, \ldots, T$ from

$$
\hat{\psi}(u, n)=\inf _{\theta} \psi_{\theta}(u, n)
$$

where, for a given value of $\theta, \psi_{\theta}(u, n)$ is defined as

$$
\begin{aligned}
\psi_{\theta}(u, n)= & \psi_{\theta}(u, 1)+\int_{0}^{u+c_{\theta}} g_{\theta}(x, 1) \hat{\psi}\left(u+c_{\theta}-x, n-1\right) d x \\
& +e^{-\lambda} \hat{\psi}\left(u+c_{\theta}, n-1\right) .
\end{aligned}
$$

Formula (3.2) is obtained by considering possible aggregate claim amounts in the first year, when the level of reinsurance is $\theta$, and the final term allows for the case of no claims in the year. 


\subsection{Continuous time}

We now consider the problem of minimising the probability of ruin in continuous time. We will need the following two quantities, defined for a given reinsurance arrangement, $\theta$. First we define $\Delta_{\theta}(u, t, y)$ to be the probability that ruin does not occur in $[0, t]$ and that the surplus at time $t$ is greater than $y$. Second,

$$
\delta_{\theta}(u, t, y) \stackrel{\text { def }}{=}-\frac{d}{d y} \Delta_{\theta}(u, t, y) .
$$

Intuitively, $\delta_{\theta}(u, t, y) d y$ is the probability that, starting from initial surplus $u$, ruin does not occur before time $t$ and the surplus at time $t$ is between $y$ and $y+d y$. At the end of this section, we will derive a formula for $\delta_{\theta}(u, t, y)$ in terms of known quantities.

The first step towards minimising the probability of ruin in continuous time is to calculate $\psi_{\theta}(u, 1)$, which we can do using Prabhu's (1961) formulae. Writing $\delta_{\theta}(u, t)=1-\psi_{\theta}(u, t)$, the required formulae are

$$
\delta_{\theta}(u, 1)=G_{\theta}\left(u+c_{\theta}, 1\right)-c_{\theta} \int_{0}^{1} \delta_{\theta}(0,1-s) g_{\theta}\left(u+c_{\theta} s, s\right) d s
$$

and for $0<t \leq 1$,

$$
\delta_{\theta}(0, t)=\frac{1}{c_{\theta} t} \int_{0}^{c_{\theta} t} G_{\theta}(y, t) d y .
$$

Then for $n>1$ we have

$$
\hat{\psi}(u, n)=\inf _{\theta} \psi_{\theta}(u, n),
$$

where, for a given value of $\theta$,

$$
\psi_{\theta}(u, n)=\psi_{\theta}(u, 1)+E(\theta)
$$

where, intuitively, $E(\theta)$ is the sum over all values of $y \in\left[0, u+c_{\theta}\right]$ of the product of (a) the probability that the surplus at time 1 is between $y$ and $y+d y$, and ruin did not occur between times 0 and 1 , and (b) $\hat{\psi}(y, n-1)$, the optimal probability of ruin between times 1 and $n$ starting at time 1 from initial surplus $y$. There are three cases to consider, depending on the value of $y$.

(1) $y=u+c_{\theta}$ : In this case the probability that the surplus is at $y$ at time 1 and ruin did not occur is $e^{-\lambda}$. Hence, the contribution to $E(\theta)$ is:

$$
e^{-\lambda} \hat{\psi}\left(u+c_{\theta}, n-1\right)
$$

(2) $y \in\left[0, c_{\theta}\right):$ In this case the contribution to $E(\theta)$ is:

$$
\int_{0}^{c_{\theta}} \delta_{\theta}(u, 1, y) \hat{\psi}(y, n-1) d y
$$


since the density associated with non-ruin in the first year with a surplus of $y$ at time 1 is $\delta_{\theta}(u, 1, y)$.

(3) $y \in\left[c_{\theta}, u+c_{\theta}\right)$ : The density associated with a surplus of $y$ at time 1 is $g_{\theta}\left(u+c_{\theta}-y, 1\right)$ since ruin could not have occurred in the first year if the surplus at time 1 is in this range. Hence the contribution to $E(\theta)$ is:

$$
\int_{c_{\theta}}^{u+c_{\theta}} g_{\theta}\left(u+c_{\theta}-y, 1\right) \hat{\psi}(y, n-1) d y
$$

Summing (3.6), (3.7) and (3.8) and substituting into (3.5), we have the following formula for $\psi_{\theta}(u, n)$ :

$$
\begin{aligned}
\psi_{\theta}(u, n)= & \psi_{\theta}(u, 1)+e^{-\lambda} \hat{\psi}\left(u+c_{\theta}, n-1\right) \\
& +\int_{0}^{c_{\theta}} \delta_{\theta}(u, 1, y) \hat{\psi}(y, n-1) d y \\
& +\int_{c_{\theta}}^{u+c_{\theta}} g_{\theta}\left(u+c_{\theta}-y, 1\right) \hat{\psi}(y, n-1) d y .
\end{aligned}
$$

We can derive a formula for $\delta_{\theta}(u, 1, y)$ by noting that by definition

$$
\Delta_{\theta}\left(0, y / c_{\theta}, y\right)=0,
$$

and for $t>y / c_{\theta}$ :

$$
\begin{gathered}
\Delta_{\theta}(u, t, y) \geq \operatorname{Pr}(\text { no claims in }[0, t])=\exp (-\lambda t), \\
\lim _{t \downarrow y / c_{\theta}} \Delta_{\theta}(u, t, y)=\exp \left(-\lambda y / c_{\theta}\right), \\
\delta_{\theta}(0, t, y)=\frac{y}{c_{\theta} t} g_{\theta}\left(c_{\theta} t-y, t\right), \\
\Delta_{\theta}(u, 1, y)=G_{\theta}\left(u+c_{\theta}-y, 1\right)-c_{\theta} \int_{0}^{1-y / c_{\theta}} g_{\theta}\left(u+c_{\theta} s, s\right) \Delta_{\theta}(0,1-s, y) d s .
\end{gathered}
$$

Formulae (3.11) and (3.12) correspond to formulae (2.1) and (2.13) on pages 112 and 114 of Gerber (1979).

Differentiating $\Delta_{\theta}(u, 1, y)$ with respect to $y$ gives:

$$
\begin{aligned}
& \frac{d}{d y} \Delta_{\theta}(u, 1, y)= \\
& -g_{\theta}\left(u+c_{\theta}-y, 1\right)-c_{\theta}\left(-1 / c_{\theta}\right) \lim _{s \uparrow 1-y / c_{\theta}} g_{\theta}\left(u+c_{\theta} s, s\right) \Delta_{\theta}(0,1-s, y) \\
& \quad+c_{\theta} \int_{0}^{1-y / c_{\theta}} g_{\theta}\left(u+c_{\theta} s, s\right) \delta_{\theta}(0,1-s, y) d s \\
& =-g_{\theta}\left(u+c_{\theta}-y, 1\right)+\lim _{1-s \downarrow y / c_{\theta}} g_{\theta}\left(u+c_{\theta} s, s\right) \Delta_{\theta}(0,1-s, y) \\
& \quad+c_{\theta} \int_{0}^{1-y / c_{\theta}} g_{\theta}\left(u+c_{\theta} s, s\right) \delta(0,1-s, y) d s
\end{aligned}
$$


Hence, using (3.10):

$$
\begin{aligned}
\delta_{\theta}(u, 1, y)= & g_{\theta}\left(u+c_{\theta}-y, 1\right)-g_{\theta}\left(u+c_{\theta}-y, 1-y / c_{\theta}\right) \exp \left(-\lambda y / c_{\theta}\right) \\
& -c_{\theta} \int_{0}^{1-y / c_{\theta}} g_{\theta}\left(u+c_{\theta} s, s\right) \delta_{\theta}(0,1-s, y) d s .
\end{aligned}
$$

For related discussions, see De Vylder and Goovaerts (1999) and Wu et al. (2003).

\subsection{Computational issues}

For small expected numbers of claims per annum, it would be possible to calculate $G_{\theta}(x, 1)$ and $g_{\theta}(x, 1)$ (by Panjer's recursion formula), $\delta_{\theta}(u, 1, y)$ (using (3.13)), $\psi_{\theta}(u, 1)$ in continuous time (using (3.3) and (3.4)) and hence $\hat{\psi}(u, T)$ (using (3.9)). For large expected numbers of claims, this is impractical and some approximate method must be used.

\section{Distribution APPROXIMATION}

In this section we show how the formulae of the previous section can be approximated by approximating the distribution of $S_{\theta}(t)$ by a translated gamma distribution. We start by introducing some notation.

Let $\alpha, \beta$ and $\kappa$ be the parameters of a translated gamma distribution with the same first three moments as $G_{\theta}(\cdot, 1)$ and let $G_{G \theta}(\cdot, t)$ and $g_{G \theta}(\cdot, t)$ be the cdf and pdf, respectively, of a gamma distribution with parameters $\alpha t$ and $\beta$. Then $G_{G \theta}(y-\kappa t, t)$ and $g_{G \theta}(y-\kappa t, t)$ approximate $G_{\theta}(y, t)$ and $g_{\theta}(y, t)$, respectively.

Note that since $\alpha, \beta>0$ and since $\alpha / \beta+\kappa=E\left[S_{\theta}(1)\right]$, we have

$$
\kappa<E\left[S_{\theta}(1)\right] \text {. }
$$

Hence, $\kappa<c_{\theta}$ if $E\left[S_{\theta}(1)\right]<c_{\theta}$, which is the net profit condition for reinsurance arrangement $\theta$, and we have restricted admissible arrangements $\theta$ to those satisfying this condition. In the following sections, we therefore know that $\kappa<c_{\theta}$.

\subsection{Discrete time}

In this section and in Section 5, we consider a fixed value of $\theta$ and we will drop the subscript $\theta$ in connection with the approximating distributions. Formulae (3.1) and (3.2) are replaced/approximated by

$$
\hat{\psi}(u, 1)=\inf _{\theta}\left(1-G_{G \theta}\left(u+c_{\theta}-\kappa, 1\right)\right)
$$

and 


$$
\begin{aligned}
\psi_{\theta}(u, n)= & 1-G_{G \theta}\left(u+c_{\theta}-\kappa, 1\right) \\
& +\int_{0}^{u+c_{\theta}} g_{G \theta}\left(u+c_{\theta}-\kappa-x, 1\right) \hat{\psi}(x, n-1) d x \\
& +G_{G \theta}(-\kappa, 1) \hat{\psi}\left(u+c_{\theta}, n-1\right) .
\end{aligned}
$$

\subsection{Continuous time}

Formulae (3.3) and (3.4) are replaced/approximated by

$$
\delta_{\theta}(u, 1)=G_{G \theta}\left(u+c_{\theta}-\kappa, 1\right)-c_{\theta} \int_{0}^{1} \delta_{\theta}(0,1-s) g_{G \theta}\left(u+\left(c_{\theta}-\kappa\right) s, s\right) d s
$$

and

$$
\delta_{\theta}(0, t)=\frac{1}{c_{\theta} t} \int_{0}^{c_{\theta} t} G_{G \theta}(y-\kappa t, t) d y
$$

which can be written in a more helpful way for computational purposes as

$$
\begin{aligned}
\delta_{\theta}(0, t)= & \left(1-\frac{\kappa}{c_{\theta}}\right) G_{G \theta}\left(\left(c_{\theta}-\kappa\right) t, t\right)+\frac{\kappa}{c_{\theta}} G_{G \theta}(-\kappa t, t) \\
& -\frac{\alpha}{\beta c_{\theta}}\left(\hat{G}_{G \theta}\left(\left(c_{\theta}-\kappa\right) t, t\right)-\hat{G}_{G \theta}(-\kappa t, t)\right),
\end{aligned}
$$

where $\hat{G}_{G \theta}(\cdot, t)$ is the cdf of a Gamma distribution with parameters $\alpha t+1$ and $\beta$. (See Dickson and Waters (1993, p. 263).) We note that for $\kappa \geq 0$, both $G_{G \theta}(-\kappa t)$ and $\hat{G}_{G \theta}(-\kappa t)$ are zero, so that in this case formula (4.3) reduces to

$$
\begin{aligned}
\delta_{\theta}(u, 1)= & G_{G \theta}\left(u+c_{\theta}-\kappa, 1\right) \\
& -\left(c_{\theta}-\kappa\right) \int_{0}^{1} G_{G \theta}\left(\left(c_{\theta}-\kappa\right)(1-s), 1-s\right) g_{G \theta}\left(u+\left(c_{\theta}-\kappa\right) s, s\right) d s \\
& +\frac{\alpha}{\beta} \int_{0}^{1} \hat{G}_{G \theta}\left(\left(c_{\theta}-\kappa\right)(1-s), 1-s\right) g_{G \theta}\left(u+\left(c_{\theta}-\kappa\right) s, s\right) d s .
\end{aligned}
$$

Similarly, formula $(3.13)$ for $\delta_{\theta}(u, 1, y)$ is replaced/approximated by

$$
\begin{aligned}
\delta_{\theta}(u, 1, y)= & g_{G \theta}\left(u+c_{\theta}-\kappa-y, 1\right) \\
& -g_{G \theta}\left(u+c_{\theta}-\kappa\left(1-y / c_{\theta}\right)-y, 1-y / c_{\theta}\right) G_{G \theta}\left(-\kappa y / c_{\theta}, y / c_{\theta}\right) \\
& -c_{\theta} \int_{0}^{1-y / c_{\theta}} g_{G \theta}\left(u+\left(c_{\theta}-\kappa\right) s, s\right) \delta_{\theta}(0,1-s, y) d s
\end{aligned}
$$

for $0 \leq y \leq c_{\theta}$, and formula (3.11) for $\delta_{\theta}(0, t, y)$ is replaced/approximated by

$$
\delta_{\theta}(0, t, y)=\frac{y}{c_{\theta} t} g_{G \theta}\left(c_{\theta} t-\kappa t-y, t\right)
$$


so that the integral in formula (4.7) becomes

$$
\int_{0}^{1-y / c_{\theta}} g_{G \theta}\left(u+\left(c_{\theta}-\kappa\right) s, s\right) \frac{y}{1-s} g_{G \theta}\left(\left(c_{\theta}-\kappa\right)(1-s)-y, 1-s\right) d s .
$$

When $\kappa \geq 0,\left(c_{\theta}-\kappa\right)(1-s)-y>0$ for $s<1-y /\left(c_{\theta}-\kappa\right)$, and so the upper limit of integration in (4.9) can be reduced to $1-y /\left(c_{\theta}-\kappa\right)$. Thus, the integral in (4.9) is zero if $y>c_{\theta}-\kappa$.

This gives us all the components required to apply formula (3.9).

\section{Process APPRoXimation}

The idea now is that each year the aggregate claims process is approximated by a translated gamma process. Thus, for $0<t \leq 1, S_{\theta}(t)$ is approximated by $Y(t)+\kappa t$, where $Y(t)$ has distribution function $G_{G \theta}$ (as in the previous section) and the parameters $\alpha, \beta$ and $\kappa$ are the same as before. The surplus process is then approximated by $u+\left(c_{\theta}-\kappa\right) t-Y(t)$, so we are effectively considering ruin when the premium rate is $c_{\theta}-\kappa$ and the aggregate claims process is a gamma process.

\subsection{Discrete time}

Under this approach the formula replacing/approximating formula (3.1) is just the same as formula (4.1), while formula (3.2) is replaced/approximated by

$$
\begin{aligned}
\psi_{\theta}(u, n)= & 1-G_{G \theta}\left(u+c_{\theta}-\kappa, 1\right) \\
& +\int_{0}^{u+c_{\theta}-\kappa} g_{G \theta}\left(u+c_{\theta}-\kappa-x, 1\right) \hat{\psi}(x, n-1) d x
\end{aligned}
$$

since for a (translated) Gamma process, the probability of no claims in any nonzero time interval is zero. A simple change of variable transforms (5.1) to

$$
\begin{aligned}
\psi_{\theta}(u, n)= & 1-G_{G \theta}\left(u+c_{\theta}-\kappa, 1\right) \\
& +\int_{\kappa}^{u+c_{\theta}} g_{G \theta}\left(u+c_{\theta}-x, 1\right) \hat{\psi}(x-\kappa, n-1) d x .
\end{aligned}
$$

We remark that formula (5.2) is equivalent to formula (4.2) when $\kappa \geq 0$, since then $g_{G \theta}(x-\kappa, 1) \equiv 0$ for $x<\kappa$, but they are not equivalent when $\kappa<0$. Thus, for $\kappa \geq 0$ we will obtain the same optimal strategy under both the distribution and the process approximations.

\subsection{Continuous time}

In this case, formulae (3.3) and (3.4) are respectively replaced/approximated by 


$$
\begin{aligned}
\delta_{\theta}(u, 1)= & G_{G \theta}\left(u+c_{\theta}-\kappa, 1\right) \\
& -\left(c_{\theta}-\kappa\right) \int_{0}^{1} \delta_{\theta}(0,1-s) g_{G \theta}\left(u+\left(c_{\theta}-\kappa\right) s, s\right) d s
\end{aligned}
$$

and

$$
\delta_{\theta}(0, t)=\frac{1}{\left(c_{\theta}-\kappa\right) t} \int_{0}^{\left(c_{\theta}-\kappa\right) t} G_{G \theta}(y, t) d y,
$$

which can be evaluated as

$$
\delta_{\theta}(0, t)=G_{G \theta}\left(\left(c_{\theta}-\kappa\right) t, t\right)-\frac{\alpha}{\beta\left(c_{\theta}-\kappa\right)} \hat{G}_{G \theta}\left(\left(c_{\theta}-\kappa\right) t, t\right)
$$

where $\hat{G}_{G \theta}$ is as defined in Section 4.2. (See Dickson and Waters (1993).) With this expression, formula (5.3) becomes

$$
\begin{aligned}
\delta_{\theta}(u, 1)= & G_{G \theta}\left(u+c_{\theta}-\kappa, 1\right) \\
& -\left(c_{\theta}-\kappa\right) \int_{0}^{1} G_{G \theta}\left(\left(c_{\theta}-\kappa\right)(1-s), 1-s\right) g_{G \theta}\left(u+\left(c_{\theta}-\kappa\right) s, s\right) d s \\
& +\frac{\alpha}{\beta} \int_{0}^{1} \hat{G}_{G \theta}\left(\left(c_{\theta}-\kappa\right)(1-s), 1-s\right) g_{G \theta}\left(u+\left(c_{\theta}-\kappa\right) s, s\right) d s,
\end{aligned}
$$

which is exactly the same as formula (4.6).

Next, formula (3.13) for $\delta_{\theta}(u, 1, y)$ is replaced/approximated by

$$
\begin{aligned}
\delta_{\theta}(u, 1, y)= & g_{G \theta}\left(u+c_{\theta}-\kappa-y, 1\right) \\
& -\left(c_{\theta}-\kappa\right) \int_{0}^{1-y /\left(c_{\theta}-\kappa\right)} g_{G \theta}\left(u+\left(c_{\theta}-\kappa\right) s, s\right) \delta_{\theta}(0,1-s, y) d s
\end{aligned}
$$

for $0 \leq y \leq c_{\theta}-\kappa$, and formula (3.11) for $\delta_{\theta}(0,1, y)$ is replaced/approximated by

$$
\delta_{\theta}(0, t, y)=\frac{y}{\left(c_{\theta}-\kappa\right) t} g_{G \theta}\left(\left(c_{\theta}-\kappa\right) t-y, t\right) .
$$

Thus, the integral in formula (5.5) becomes

$$
\int_{0}^{1-y /\left(c_{\theta}-\kappa\right)} g_{G \theta}\left(u+\left(c_{\theta}-\kappa\right) s, s\right) \frac{y}{1-s} g_{G \theta}\left(\left(c_{\theta}-\kappa\right)(1-s)-y, 1-s\right) d s .
$$

We remark that formula (4.9) reduces to this when $\kappa \geq 0$.

Thus, we have all the components required to apply formula (3.9), and we note that for $\kappa \geq 0$, we will obtain the same results under this approach as under the approach of Section 4.2, provided that $u>0$. 


\section{Computational Procedures}

For any realistic model for aggregate claims, the only feasible approach to applying the formulae in the previous three sections is a numerical one. In this section we describe our approach to calculations.

\subsection{Numerical integration}

Before describing how we performed numerical integration, it is useful to present the context of our calculations. In each of the examples in the next section, we are working with an aggregate claims process for which the expected aggregate claim amount per annum before reinsurance is 100 . Thus, if we consider the integrals in formulae (4.2) and (5.1), we see that the range of integration is sufficiently large that we should choose a method of numerical integration that is a compromise between accuracy and computer run time (which can be considerable in the case of continuous time ruin). For formula (4.2), we performed numerical integration over the interval $\left[0, u+c_{\theta}\right]$ by applying the trapezoidal rule using intervals of length 0.1 , with a simple adjustment when $u+c_{\theta}$ was not an integer multiple of 0.1 . To implement this, for $n=2,3, \ldots, 10$, we calculated values of $\hat{\psi}(x, n-1)$ for values of $x$ that were integer multiples of 0.1 , and used linear interpolation to give values of $\hat{\psi}(x, n-1)$ if $x$ was not an integer multiple of 0.1. A similar approach was applied to formula (5.1). However, for each of these formulae, it was often possible to reduce the range of integration, as described in Section 6.2. For continuous time ruin, the integrals in formulae (4.3) and (5.3) were also calculated using the trapezoidal rule, this time using intervals of length 0.001 .

A more complicated issue arose in the calculation of (4.9), and similarly in the integral in (5.5). In formula (4.9), a singularity will arise if in the second $g_{G \theta}$ function, the first argument goes to 0 and the second argument is less than 1 . In such circumstances, numerical integration was performed by first transforming the integral to remove the singularity and using the extended midpoint rule with 1,000 intervals as described in Section 4.4 of Press et al. (1992). Otherwise, we applied the trapezoidal rule with 1,000 intervals.

\subsection{Truncation}

It is possible to reduce the amount of calculation required but still control the accuracy of our calculations by applying a truncation procedure. This idea was introduced by De Vylder and Goovaerts (1988) who calculated finite time ruin probabilities for a discrete time risk model. In what follows we state without proof results for continuous time ruin probabilities for the original model, but these results equally apply in discrete time. The results can be proved by applying ideas in De Vylder and Goovaerts (1988). 
For given values of $u$ and $\theta$, we can calculate $\psi_{\theta}(u, 1)$. Let us now choose a small positive quantity, $\varepsilon$, and define

$$
\psi_{\theta}^{*}(u, 1)=\left\{\begin{array}{l}
\psi_{\theta}(u, 1) \text { if this value is at least } \varepsilon \\
0 \text { otherwise }
\end{array}\right.
$$

and define

$$
\hat{\psi}^{*}(u, 1)=\inf _{\theta} \psi_{\theta}^{*}(u, 1) .
$$

Then for all $u \geq 0$,

$$
\begin{aligned}
& 0 \leq \psi_{\theta}(u, 1)-\psi_{\theta}^{*}(u, 1) \leq \varepsilon, \\
& 0 \leq \hat{\psi}(u, 1)-\hat{\psi}^{*}(u, 1) \leq \varepsilon .
\end{aligned}
$$

Next, for $n=2,3,4, \ldots$ we adapt equation (3.9) and define

$$
\begin{aligned}
A_{\theta}(u, n)= & \psi_{\theta}^{*}(u, 1)+e^{-\lambda} \hat{\psi}^{*}\left(u+c_{\theta}, n-1\right) \\
& +\int_{0}^{c_{\theta}} \delta_{\theta}(u, 1, y) \hat{\psi}^{*}(y, n-1) d y \\
& +\int_{c_{\theta}}^{u+c_{\theta}} g_{\theta}\left(u+c_{\theta}-y, 1\right) \hat{\psi}^{*}(y, n-1) d y .
\end{aligned}
$$

Now define

$$
\psi_{\theta}^{*}(u, n)=\left\{\begin{array}{l}
A_{\theta}(u, n) \text { if this value is at least } \varepsilon \\
0 \text { otherwise }
\end{array}\right.
$$

and define

$$
\hat{\psi}^{*}(u, n)=\inf _{\theta} \psi_{\theta}^{*}(u, n) .
$$

Then for all $u \geq 0$,

$$
\begin{aligned}
& 0 \leq \psi_{\theta}(u, n)-\psi_{\theta}^{*}(u, n) \leq(2 n-1) \varepsilon, \\
& 0 \leq \hat{\psi}(u, n)-\hat{\psi}^{*}(u, n) \leq(2 n-1) \varepsilon .
\end{aligned}
$$

\subsection{Finding optimal retention levels}

Our method of finding optimal retention levels was to use a grid search. For example, in the case of proportional reinsurance where we denote the retained proportion $a$, for each required value of $u$, we first calculated values of $\psi_{\theta}(u, 1)$ for each admissible retention level that was an integer multiple of 0.01 . We then selected $\hat{\psi}(u, 1)$ as the minimum of this set of ruin probabilities. Values of $\psi_{\theta}(u, n)$ 
were calculated over the same set of retention levels for $n=2,3, \ldots, T$, and, for each value of $n, \hat{\psi}(u, n)$ was calculated as the minimum of this set of ruin probabilities. In the case of excess loss reinsurance, where we denote the retention level as $M$, we followed the same approach, this time limiting the calculations to the set of admissible retention levels that were integer multiples of 0.1 , with a maximum retention of 10 (which is 10 times the mean individual claim amount in each of the examples in the following section). This method of finding optimal retention levels can be refined as required, but such refinement comes at a computational cost, and as our aim is to illustrate the effect of a dynamic reinsurance policy, we did not consider it necessary to consider larger sets of retention levels.

\section{NumericAl EXAMPLeS}

Example 7.1. In this example, individual claim amounts have an exponential distribution with mean 1 and the Poisson parameter, $\lambda$, is 100. The premium loading factors are $10 \%$ for the insurer and $20 \%$ for the reinsurer. We consider first excess loss reinsurance and ruin in discrete time. We use the translated gamma distribution approximation, as described in Section 4, and our algorithms incorporate a truncation parameter, $\varepsilon=0.5 \times 10^{-7}$, as described in Section 6. The insurer's initial surplus is 23 , which has been chosen so that, without any reinsurance, the probability of ruin within 10 years is about 5\% (in fact, 0.0520)

Using the formulae and procedures in Sections 4 and 6, we can show that the optimal strategy for this insurer is to set $M=1.5$ in the first year. If the insurer continues to follow the optimal strategy each year, the probability of ruin within 10 years, $\hat{\psi}(23,10)$, will be 0.0260 , a reduction of around $50 \%$. With an excess loss retention level of 1.5, the insurer's net income is 83.2 and the net profit has expected value 5.5 and standard deviation 9.4.

Table 7.1 shows for each remaining year and for selected values of the insurer's surplus, the optimal excess loss retention for the coming year, $M$, and the corresponding optimal probability of ruin, $\hat{\psi}(u, t)$, assuming the optimal strategy is adopted every year. For example, suppose that at the end of the first year, the insurer's surplus has increased from 23 to 25 . The remaining term is $t=9$ years and the entries in Table 7.1 show that the optimal strategy now is to set the excess loss retention level for the coming year to $M=1.4$, with the (optimal) probability of ruin within 9 years now being 0.0193. If the insurer's surplus had dropped to 15, the optimal strategy would have been to set $M=1.6$, so that we would have $\hat{\psi}(15,9)=0.0705$. If, at the end of 6 years, the insurer's surplus has reached 40 , the optimal strategy would be to set $M=0.7(\hat{\psi}(40,4)=0.0000)$. Note that a reinsurance arrangement is admissible only if the insurer's net income exceeds its net expected claims. For the choice of claim distribution and premium loadings in this example, the smallest possible excess loss retention level is $M=0.7$.

It can be seen from Table 7.1 that, for a fixed current surplus $u, \hat{\psi}(u, t)$ is an increasing function of the remaining term $t$, as it must be. Similarly, for fixed 
TABLE 7.1.

OPTIMAL STRATEGY: EXPONENTIAL CLAIMS, LOADINGS 10\%/20\%, EXCESS LOSS, DISCRETE TIME RUIN, DISTRIBUTIONAL APPROXIMATION.

\begin{tabular}{c|c|ccccccccc}
\hline \hline \multirow{2}{*}{$\begin{array}{c}\text { Surplus, } \\
\boldsymbol{u}\end{array}$} & & \multicolumn{8}{|c}{ Remaining term, $\boldsymbol{t}$} \\
& & $\mathbf{9}$ & $\mathbf{8}$ & $\mathbf{7}$ & $\mathbf{6}$ & $\mathbf{5}$ & $\mathbf{4}$ & $\mathbf{3}$ & $\mathbf{2}$ & $\mathbf{1}$ \\
\hline \multirow{2}{*}{5} & $M$ & 3.4 & 3.4 & 3.3 & 3.3 & 3.2 & 3.1 & 2.9 & 2.6 & 1.9 \\
& $\hat{\psi}(u, t)$ & 0.2094 & 0.2078 & 0.2057 & 0.2028 & 0.1986 & 0.1923 & 0.1826 & 0.1661 & 0.1307 \\
10 & $M$ & 2.1 & 2.1 & 2.1 & 2.0 & 2.0 & 1.8 & 1.7 & 1.3 & 0.7 \\
& $\hat{\psi}(u, t)$ & 0.1278 & 0.1262 & 0.1240 & 0.1208 & 0.1164 & 0.1097 & 0.0993 & 0.0814 & 0.0390 \\
15 & $M$ & 1.6 & 1.6 & 1.5 & 1.5 & 1.4 & 1.3 & 1.1 & 0.8 & 0.7 \\
& $\hat{4}(u, t)$ & 0.0705 & 0.0689 & 0.0667 & 0.0636 & 0.0592 & 0.0528 & 0.0429 & 0.0264 & 0.0048 \\
20 & $M$ & 1.4 & 1.4 & 1.4 & 1.3 & 1.3 & 1.1 & 1.0 & 0.7 & 0.7 \\
& $\hat{\psi}(u, t)$ & 0.0371 & 0.0357 & 0.0339 & 0.0313 & 0.0277 & 0.0227 & 0.0155 & 0.0060 & 0.0004 \\
25 & $M$ & 1.4 & 1.4 & 1.4 & 1.3 & 1.2 & 1.0 & 0.8 & 0.7 & 0.7 \\
& $\hat{\psi}(u, t)$ & 0.0193 & 0.0182 & 0.0168 & 0.0148 & 0.0123 & 0.0089 & 0.0047 & 0.0010 & 0.0000 \\
30 & $M$ & 1.4 & 1.4 & 1.3 & 1.2 & 1.1 & 0.9 & 0.7 & 0.7 & 0.7 \\
& $\hat{\psi}(u, t)$ & 0.0098 & 0.0090 & 0.0080 & 0.0067 & 0.0051 & 0.0031 & 0.0011 & 0.0001 & 0.0000 \\
35 & $M$ & 1.4 & 1.3 & 1.2 & 1.1 & 1.0 & 0.8 & 0.7 & 0.7 & 0.7 \\
& $\hat{\psi}(u, t)$ & 0.0049 & 0.0044 & 0.0037 & 0.0029 & 0.0019 & 0.0009 & 0.0002 & 0.0000 & 0.0000 \\
40 & $M$ & 1.3 & 1.2 & 1.2 & 1.0 & 0.8 & 0.7 & 0.7 & 0.7 & 0.7 \\
& $\hat{\psi}(u, t)$ & 0.0024 & 0.0020 & 0.0016 & 0.0011 & 0.0006 & 0.0002 & 0.0000 & 0.0000 & 0.0000 \\
\hline \hline
\end{tabular}

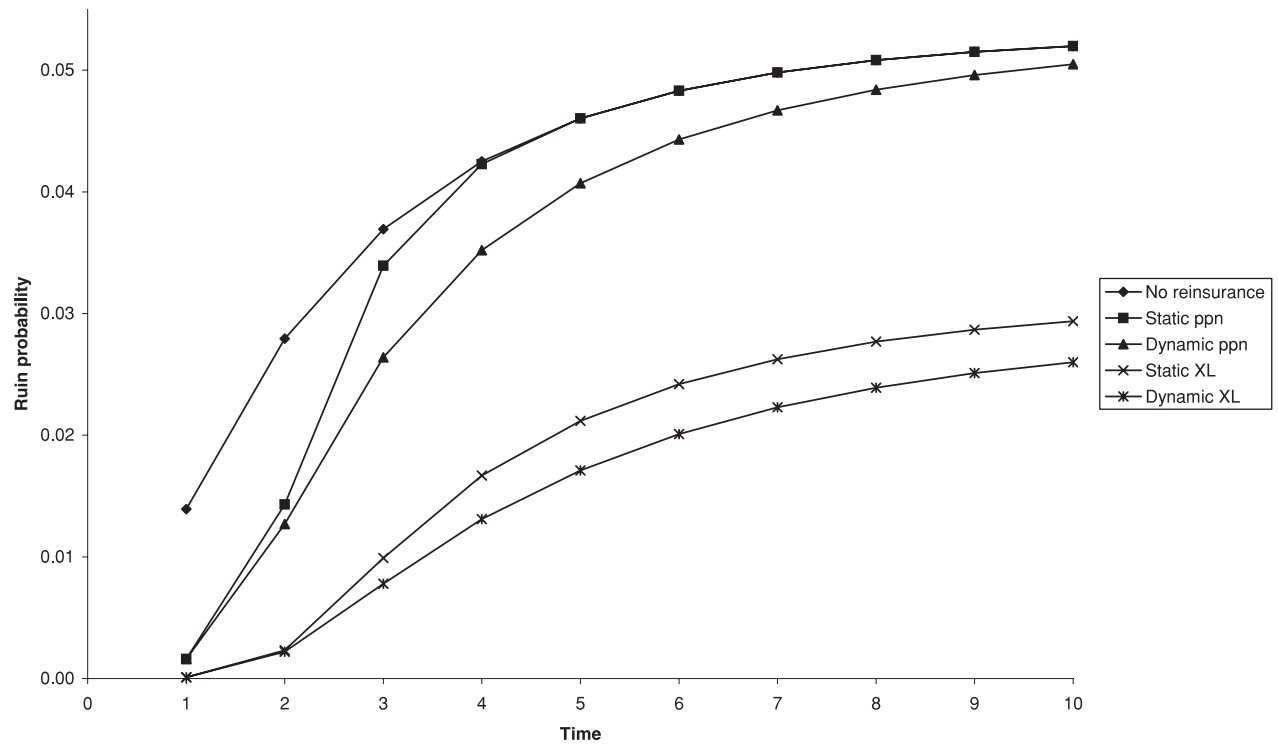

FIGURE 7.1: Discrete time ruin probabilities under static and dynamic strategies, exponential claims. 
remaining term $t, \hat{\psi}(u, t)$ is a decreasing function of the current surplus $u$, as it must be. Another feature of Table 7.1 is that the optimal retention, $M$, is an increasing function of the remaining time $t$, for a fixed current surplus $u$, and a decreasing function of the current surplus $u$ for a fixed remaining time $t$.

Figure 7.1 illustrates discrete time ruin probabilities under both static and dynamic reinsurance strategies for both proportional and excess loss reinsurance for time horizons $t=1,2, \ldots, 10$ and $u=23$. Under a static strategy, we choose the retention level ( $a$ or $M$ ) which, when applied each year, minimises the probability of ruin over a fixed period. For example, in the case of excess loss reinsurance the optimal static strategy is to choose $M=1.3$ each year if we wish to minimise the ruin probability over a five year time period, but if we wish to minimise the ruin probability over a ten year period, the optimal static strategy is to choose $M=1.6$ for each of the ten years. We can find the optimal level of reinsurance under a static strategy by applying the same grid search as described in Section 6.3, and we can calculate ruin probabilities by applying a formula similar to (4.2), the difference being that $\hat{\psi}$ in formula (4.2) is replaced by $\psi_{\theta}$. We observe from Figure 7.1 that, as we would expect, excess loss reinsurance reduces the ruin probability over a ten year period by a greater amount than proportional reinsurance, and that a dynamic reinsurance policy is better than a static one, reducing the ten year probability of ruin by around $4 \%$ in the case of proportional reinsurance (0.0520 goes down to 0.0498) and by nearly $13 \%$ in the case of excess loss reinsurance (0.0294 goes down to 0.0257). We also calculated that for $t=4,5, \ldots$, 10 , the optimal static strategy under proportional reinsurance is to set $a=1$, so that the minimum ruin probabilities for these values of $t$ are the same as the ruin probabilities with no reinsurance.

Finally, we remark that although the results presented in Table 7.1 and Figure 7.1 have been calculated using the methodology presented in Section 4, we could equally have used the methodology presented in Section 5. Although the results obtained are not identical (as the translated gamma parameter $\kappa$ is negative for each excess loss retention considered), the numerical results for minimum ruin probabilities are the same to four decimal places, and the optimal strategies are the same.

Example 7.2. In this example we consider continuous time ruin. We let the individual claim amount distribution be Pareto(4,3) (so that the mean is 1) and, as in Example 7.1, we set the Poisson parameter, $\lambda$, to be 100. The insurer's initial surplus is 49, so that without reinsurance the probability of ruin within 10 years is 0.0504 . For proportional reinsurance, the premium loading factors are $10 \%$ and $20 \%$ for the insurer and the reinsurer, respectively, while for excess loss reinsurance we have considered both this scenario and the case when the reinsurer's loading factor is $30 \%$. As in the previous example, the truncation parameter is $\varepsilon=0.5 \times 10^{-7}$.

We again consider both proportional and excess loss reinsurance, and consider both static and dynamic reinsurance strategies.

Figure 7.2 shows ruin probabilities under both static and dynamic reinsurance strategies, for both proportional and excess loss reinsurance, and without reinsurance. 


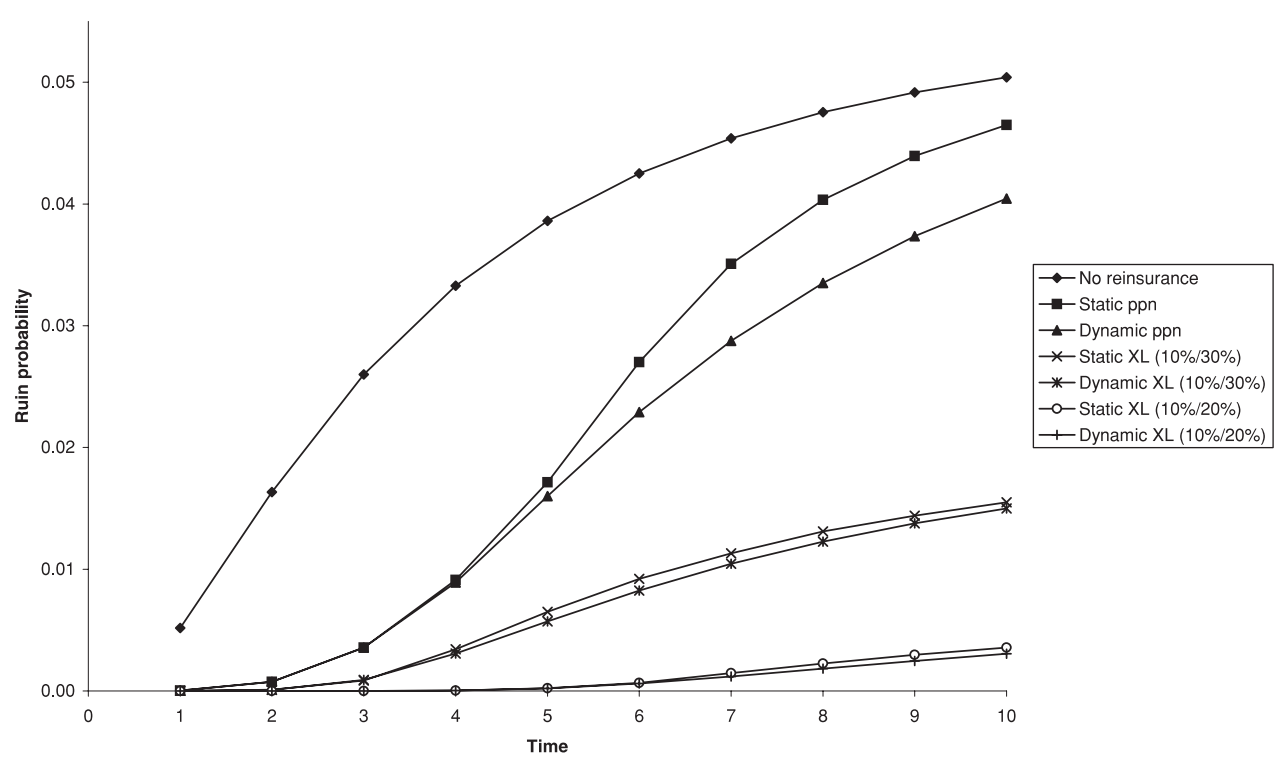

FIgURE 7.2: Continuous time ruin probabilities under static and dynamic strategies, Pareto claims.

In the case of proportional reinsurance, it is easy to calculate that, as a function of $a$, we have $\kappa=100 a / 3>0$, so that the approaches of Sections 4.2 and 5.2 yield the same results (and so too in the case of no reinsurance, where $a=1$ ). In the case of excess loss reinsurance, the approaches of Sections 4.2 and 5.2 yield the same optimal static and dynamic strategies, but give very slightly different values for optimal ruin probabilities (differences of no more than 1 in the fourth decimal place).

Looking at Figure 7.2, two points are immediately obvious. First, the dynamic strategy is better than the static strategy for each type of reinsurance, although it is not much better in the case of excess loss reinsurance. Second, as we might expect, even with a dynamic strategy, proportional reinsurance does not reduce the insurer's ruin probability nearly as much as excess loss reinsurance does. We remark that we also observed both these features when the individual claim amount distribution is exponential, but we have not graphed these results.

\section{Conclusions}

In Example 7.1 we observed from Table 7.1 that the optimal excess loss retention was a decreasing function of the insurer's surplus for a given remaining time. This may seem counter-intuitive - we might expect an increase in the insurer's financial strength to result in a higher retention level. This feature arises because we have chosen to minimise the insurer's probability of ruin up 
to a fixed time point. Given this criterion, it can be optimal for an insurer with a large current surplus to reinsure as much as possible of its portfolio; the expected surplus at the end of the time limit will be reduced, but, by reducing the variability of its net claims, its probability of ruin will also be reduced. Conversely, it may be optimal for an insurer with a small current surplus to reinsure relatively little of its portfolio since, with a larger expected net surplus, it will stand the best chance of increasing its surplus and hence reducing its probability of ruin over the remaining term. We could adopt a different optimality criterion. For example, for a given current surplus and a given remaining time, we could choose the retention level which gives the highest expected net surplus, subject to the probability of ruin being no higher than a specified value. Alternatively, we could have a rolling time horizon rather than a fixed time horizon. For example, referring back to Example 7.1 and assuming that the insurer has current surplus $u=20$ and has a five year time horizon, we can see from Table 7.1 that the optimal excess loss retention is $M=1.3$. Now suppose that after one year the insurer's surplus is 25 . Using a fixed time horizon, as in Example 7.1 so that the remaining time is 4 years, the optimal retention is now $M=1.0$. However, if the insurer uses a rolling five year horizon, so that the time horizon is reset to five years at the end of every year, the optimal retention is $M=1.2$. A rolling horizon criterion has some intuitive appeal but cannot lead to a lower probability of ruin over a fixed time period than the original criterion (since the original criterion was precisely to minimise this probability). Alternative optimality criteria will be investigated in a further paper.

It is computationally much more efficient to evaluate the optimal static reinsurance policy, and, as illustrated in Example 7.2, the difference in ruin probabilities under static and dynamic strategies may not be great. Ruin probabilities under a static strategy provide upper bounds to ruin probabilities under a dynamic strategy, and if ruin probabilities are sufficiently low under a static strategy, there seems to be little advantage in evaluating the optimal dynamic strategy.

One of our objectives was to investigate the differences between what we have termed the translated gamma distribution and process approximations. In the context of our particular study, we have shown that:

(a) these two approximations are equivalent when $\kappa$ is greater than zero, and

(b) when $\kappa$ is negative, the numerical results from the two approximations are almost identical in both our examples in Section 7.

\section{REFERENCES}

Centeno, M. DE L. (1986) Measuring the effects of reinsurance by the adjustment coefficient. Insurance: Mathematics \& Economics 5, 169-182.

Daykin, C.D., Pentikäinen, T. and Pesonen, M. (1993) Practical Risk Theory for Actuaries. Chapman and Hall, London.

De Vylder, F. and Goovaerts, M.J. (1988) Recursive calculation of finite time ruin probabilities. Insurance: Mathematics \& Economics 7, 1-8. 
De Vylder, F. and Goovaerts, M.J. (1999) Explicit finite-time and infinite-time ruin probabilities in the continuous case. Insurance: Mathematics \& Economics 24, 155-172.

DiCKSON, D.C.M. and WATERS, H.R. (1993) Gamma processes and finite time survival probabilities. ASTIN Bulletin 23, 259-272.

Dickson, D.C.M. and Waters, H.R. (1996) Reinsurance and ruin. Insurance: Mathematics \& Economics 19, 61-80.

Gerber, H.U. (1979) An Introduction to Mathematical Risk Theory. S.S. Huebner Foundation, Philadelphia, PA.

Hipp, C. and Vogt, M. (2003) Optimal dynamic XL reinsurance. ASTIN Bulletin 33, 193-207.

Prabhu, N.U. (1961) On the ruin problem of collective risk theory. Annals of Mathematical Statistics 32, 757-764.

Press, W.H., Teukolsky, S.A., Vetterling, W.T. and Flannery, B.P. (1992) Numerical Recipes in Fortran 77, 2nd edition. Cambridge University Press, Cambridge.

Promislow, S.D. and YounG, V.R. (2005) Minimizing the probability of ruin when claims follow Brownian motion with drift. North American Actuarial Journal 9(3), 109-128.

Schmidi, H. (2001) Optimal proportional reinsurance policies in a dynamic setting. Scandinavian Actuarial Journal, 55-68.

Schmidli, H. (2002) On minimising the ruin probability by investment and reinsurance. Annals of Applied Probability 12, 890-907.

SEAL, H.L. (1978) From aggregate claims distribution to probability of ruin. ASTIN Bulletin 10, 47-53.

WU, R., WANG, G., and WEI, L. (2003) Joint distributions of some actuarial random vectors containing the time of ruin. Insurance: Mathematics \& Economics 33, 147-161.

DAVID C.M. DiCKSON

Centre for Actuarial Studies

Department of Economics

University of Melbourne

Victoria 3010

Australia

E-mail:dcmd@unimelb.edu.au

HOWARD R. WATERS

Department of Actuarial Mathematics and Statistics

Heriot-Watt University

Edinburgh EH14 4AS

Great Britain

Email: H.R.Waters@ma.hw.ac.uk 